\title{
Análise de dados espaciais das deposições de resíduos sólidos da construção civil no município de Guarulhos, São Paulo
}

Spatial data analysis of disposal points of construction waste in the city of Guarulhos, São Paulo

Análisis de datos espaciales de las deposiciones de residuos sólidos de la construcción civil en el municipio de Guarulhos, São Paulo

Walter Rubens Bolitto Carvalho Estudante do curso de Engenharia Química, UNG, Brasil bolitto.walter@gmail.com

Natália Góes dos Santos Barom

Professora Mestre, UNG, Brasil. natigoes@gmail.com

Silvania Maria Netto Professora Doutora, UNG, Brasil. silvania.netto@prof.ung.br 


\section{RESUMO}

A geração de resíduos se tornou um desafio em grandes centros urbanos, cidades e países em desenvolvimento, devido ao crescente grau de urbanização e falta de planejamento urbano, fazendo com que os governos notassem a necessidade de políticas públicas para a gestão dos resíduos sólidos. Ao interpretarmos cidades como sistemas complexos, conseguimos discutir a aplicabilidade de programas governamentais, pois a grande quantidade de elementos e interagentes faz com que os indicadores sejam impactados de forma não-linear. $O$ presente trabalho apresenta um estudo multidimensional de dados espaciais no Município de Guarulhos, São Paulo, mostrando como estes fatores interferem na geração de resíduos sólidos em áreas irregulares, enfatizando aos resíduos da construção civil (RCC). Foi possível observar, através da análise de variáveis, a existência de pontos de descarte irregular de RCC na região Central do Município de Guarulhos, coincidindo com indicadores sociais mais elevados e densidade populacional. Observou-se, ainda, uma adesão parcial da população aos Pontos de Entrega Voluntária (PEVs), evidenciando a necessidade de investimentos em educação ambiental, próximas a região Central do Município, e ampliação dos instrumentos públicos nas regiões mais afastadas. Os resultados obtidos mostram que este tipo de mapeamento possibilita a análise do impacto de indicadores oferecem dados que podem explicar potenciais fatores causadores de determinados fenômenos e serem utilizados na construção de políticas públicas mais eficientes.

Palvras-chave: Gestão de Resíduos Sólidos, Resíduos da Construção Civil, Análise Exploratória de Dados Espaciais, Desigualdade Ambiental, Qualidade Ambiental.

\section{ABSTRACT}

Due the increasing degree of urbanization and lack of urban planning, waste generation becomes a challenge in large urban centers and cities in developing countries, causing governments realize the need to develop public policies related to solid waste management. In interpreting cities as complex systems, we have been able to discuss the applicability of government programs, since the large number of elements and interactors causes indicators to be impacted in a non-linear manner. The present work presents a multidimensional study of spatial data in the Municipality of Guarulhos, São Paulo, showing how these factors interfere in the generation of solid waste in irregular areas, emphasizing the construction waste (CW). It was possible to observe, through the analysis of variables, the existence of irregular points of $\mathrm{CW}$ in the Central region of the Municipality of Guarulhos, coinciding with higher social indicators and population density. It was also observed a partial adhesion of the population to the Voluntary Delivery Points (VDPs), evidencing the need for investments in environmental education, near the Central region of the Municipality, and expansion of public instruments in the most remote regions. The results show that this mapping type allows the analysis of the impact of indicators that offer data that may explain potential factors that cause certain phenomena and be used in the construction of more efficient public policies.

Keywords: Solid Waste Management, Civil Construction Waste, Exploratory Analysis of Spatial Data, Environmental Inequality, Environmental Quality.

\section{RESUMEN}

La generación de residuos se ha convertido en un desafío en grandes centros urbanos, ciudades y países en desarrollo, debido al creciente grado de urbanización y falta de planificación urbana, haciendo que los gobiernos noten la necesidad de políticas públicas para la gestión de los residuos sólidos. Al interpretar ciudades como sistemas complejos, conseguimos discutir la aplicabilidad de programas gubernamentales, pues la gran cantidad de elementos e interactivos hace que los indicadores sean impactados de forma no lineal. El presente trabajo presenta un estudio multidimensional de datos espaciales en el Municipio de Guarulhos, São Paulo, mostrando cómo estos factores interfieren en la generación de residuos sólidos en áreas irregulares, enfatizando los residuos de la construcción civil (RCC). Se pudo observar, a través del análisis de variables, la existencia de puntos de descarte irregular de RCC en la región Central del Municipio de Guarulhos, coincidiendo con indicadores sociales más elevados y densidad poblacional. Se observó, además, una adhesión parcial de la población a los Puntos de Entrega Voluntaria (PEVs), evidenciando la necesidad de inversiones en educación ambiental, próximas a la región Central del Municipio, y ampliación de los instrumentos públicos en las regiones más alejadas. Los resultados obtenidos muestran que este tipo de cartografía posibilita el análisis del impacto de indicadores ofrecen datos que pueden 
explicar potenciales factores causantes de determinados fenómenos y ser utilizados en la construcción de políticas públicas más eficientes.

Palabras Clave: Gestión de Residuos Sólidos, Residuos de la Construcción Civil, Análisis Exploratorio de Datos Espaciales, Desigualdad Ambiental, Calidad Ambiental. 


\section{Periódica Eletrônica}

\section{INTRODUÇÃO}

O processo de crescimento populacional nas últimas décadas, principalmente em centros urbanos, originou o debate relacionado ao desenvolvimento de políticas públicas na gestão dos resíduos urbanos, pois tanto a geração quanto sua destinação final, ambientalmente e sanitariamente, adequadas são um dos maiores desafios com que se defronta a sociedade contemporânea. Esta é uma preocupação mundial, sobretudo em relação aos resíduos sólidos domiciliares, cuja geração tem aumentado, o que levanta questões quanto ao seu gerenciamento inadequado, à falta de soluções e de áreas de disposição final, gerando preocupações com a degradação do meio ambiente e a saúde do ser humano (JACOBI \& BESEN, 2011).

Dentre as diferentes legislações existentes relacionadas à política ambiental em território brasileiro, a Política Nacional de Resíduos Sólidos (PNRS), instituída pela Lei no 12.305, em 2010, preencheu as lacunas existentes nas leis anteriores referentes ao lixo, e objetivou estabelecer a responsabilidade dos municípios com os resíduos produzidos em seus territórios, estipulando o princípio da responsabilidade compartilhada do ciclo de vida dos produtos, e exigindo a expansão da logística reversa, através de pontos de entrega voluntária (PEV) e coleta seletiva porta a porta (BRASIL, 2010). FERREIRA et al. (2017) observaram que o investimento em infraestrutura melhorou a gestão de resíduos nos municípios maiores, enquanto, para os municípios menores, o aumento na eficiência ocorreu através da construção de consórcios intermunicipais, por meio do trabalho conjunto entre os municípios, da mesma região, e a iniciativa privada.

Diferente da gestão de resíduo domiciliar municipal, que opera a coleta porta a porta de resíduos domiciliares, os resíduos da construção civil (RCC) exigem uma maior atenção dos municípios, devido as variáveis que influenciam sua disposição final, tais como, tipo de resíduo e tamanho de sua produção, sendo muitas vezes superiores quando comparados à geração de resíduo domiciliar (TESSARO et al, 2012). Sob a ótica de sistemas complexos, variáveis como fatores sociais e físicos, relacionados ao espaço urbano, impactam no aproveitamento de programas de reciclagem e de pontos de entrega voluntária (PEV) e devem ser exploradas pelas prefeituras, com o intuito de diminuir o lixo destinado a aterros sanitário, terrenos baldios e ruas (COSTA, 2003).

O desenvolvimento da indústria da construção civil no Brasil, ocasionou o crescimento de resíduos gerados em canteiros de obra e, muitas vezes, estes são transportados de forma irregular a córregos, esquinas e terrenos baldios, gerando não apenas depósitos irregulares de descarte como, também, novas áreas de riscos de enchentes e deslizamentos, e causando prejuízos para os municípios. A Resolução 307/2002 do CONAMA (CONAMA, 2002), estabelece as diretrizes e procedimentos para que as construtoras realizem a gestão dos resíduos produzidos em suas construções e demolições, e possibilitem a redução do impacto ambiental. No entanto, observou-se que grande parte do setor da construção civil ainda não se mobilizou no atendimento à resolução (LINHARES et al, 2007). Essa resolução também aborda a implantação de PEVs, no processo de logística reversa, sugerindo que os municípios implementem também ecopontos, destinados a entrega de pequenos volumes de resíduos de construção e demolição produzidos por munícipes e, ainda, recomenda uma melhor distribuição destes pontos para atender a população de bairros mais afastados e, assim, maior acesso à área de bacia de captação (MINISTÉRIO DO MEIO AMBIENTE, 2010).

O Município de Guarulhos, localizado na Região Metropolitana de São Paulo, com 1,2 milhões de habitantes (IBGE, 2010), em seu processo construtivo de suas políticas públicas, relacionadas à gestão dos resíduos urbanos, Guarulhos teve avanços em 2001 com a implantação dos PEVs e da Coleta Seletiva. O Decreto $n^{\circ}$ 25754, de 1 de setembro de 2008, 


\section{Periódica Eletrônica}

\section{Fórum Ambiental}

Volume 14, Número 2, 2018

da Alta Paulista

ISSN 1980-0827

regulamentou o Plano Integrado de Gerenciamento de Resíduos da Construção Civil (GUARULHOS, 2008). Em 2011 houve a criação do Plano Diretor de Resíduos Sólidos (GUARULHOS, 2011), objetivando oferecer um panorama inicial de integração entre os diversos agentes e planos de organização de ações a serem implementadas, considerando a elevada quantidade de órgãos que se envolvem diretamente na coleta do resíduo, às quais geram problemas de logística e má distribuição de funcionários para varrição e coleta pelo município (DE LÓCCO, 2017). Segundo o Plano Diretor de Resíduos Sólidos, a limpeza corretiva urbana na cidade é responsabilidade do Departamento de Limpeza Urbana (DELURB), pertencente à Secretaria de Serviços Públicos, juntamente com a empresa de economia mista Progresso e Desenvolvimento de Guarulhos S/A (PROGUARU), e que além da zeladoria, devem monitorar e fiscalizar os pontos de descarte irregular para que novos casos não se repitam no mesmo espaço, além do poder de aplicação de multa ao infrator (GUARULHOS, 2011). As medidas utilizadas para evitar o descarte irregular, em especial, devido a construção dos PEVs, assim como a fiscalização realizada, possibilitou não apenas uma melhoria urbana como, por exemplo, redução de enchentes por lixo acumulado e contaminação de rios e córregos, mas também uma diminuição nos gastos públicos relacionado à limpeza, evitando-se o custo médio de $R \$ 295,07$ por tonelada de material que é direcionado aos PEVs ao invés de ser descartado na rua (AMORIM, 2016).

O presente trabalho teve por objetivo diagnosticar quais os determinantes que se correlacionam espacialmente com os pontos de descartes, sejam estes socioeconômicos, territoriais ou referentes ao acesso à urbanidade, oferecendo uma maior compreensão sobre as variáveis que impactam na distribuição dos pontos de disposição irregular de resíduos no município de Guarulhos, oferecendo novas abordagens para as tomadas de decisão municipais no processo de gestão de resíduos.

\section{METODOLOGIA}

Através da revisão do Plano Diretor de Desenvolvimento Urbano, Econômico e Social de Guarulhos (AMBIENS, 2012), obteve-se o mapeamento das deposições irregulares e PEVs (Fig. 1), seguido da transformação do mapa no formato raster e georreferenciado, possibilitando uma contagem das variáveis por bairro. A organização e padronização dos dados foi realizada com auxílio do software-livre QGIS 2.8 .

Os dados sociodemográficos e de infraestrutura foram obtidos através do Sistema IBGE de Recuperação Automática (Tab. 1), com o objetivo de compreender o impacto do descarte clandestino de RCC na desigualdade ambiental, já que as classes sociais menos favorecidas em áreas mais afastadas da cidade sofrem os maiores riscos sociais e ambientais (VIÉGAS, 2006). 0 crescimento urbano desenfreado observado na cidade, assim como em toda Região Metropolitana de São Paulo, evidencia uma maior concentração de renda na região Central e Oeste do município, e observa-se a diminuição da renda familiar com uma maior distância do centro da cidade, evidenciando a carência econômica e urbana nas regiões periféricas da cidade (Fig. 2). Por fazerem parte de Área de Proteção Ambiental, os bairros Cabuçu de Cima e Tanque Grande ausentam dados estatísticos sociais e foi feito um recorte destes para a análise (GUARULHOS, 2010). A região Norte do município também faz parte de Área de Proteção Ambiental, mas com a presença de moradias, é caracterizada como zona rural ou semiurbanizada, sendo possível observar uma menor densidade demográfica e características distintas das outras regiões (SAAD et al, 2007). 


\section{Periódica Eletrônica}

\section{Fórum Ambiental}

Volume 14, Número 2, 2018

\section{da Alta Paulista}

ISSN 1980-0827

Figura 1: Mapa das localizações das deposições irregulares e PEVs do Município de Guarulhos.

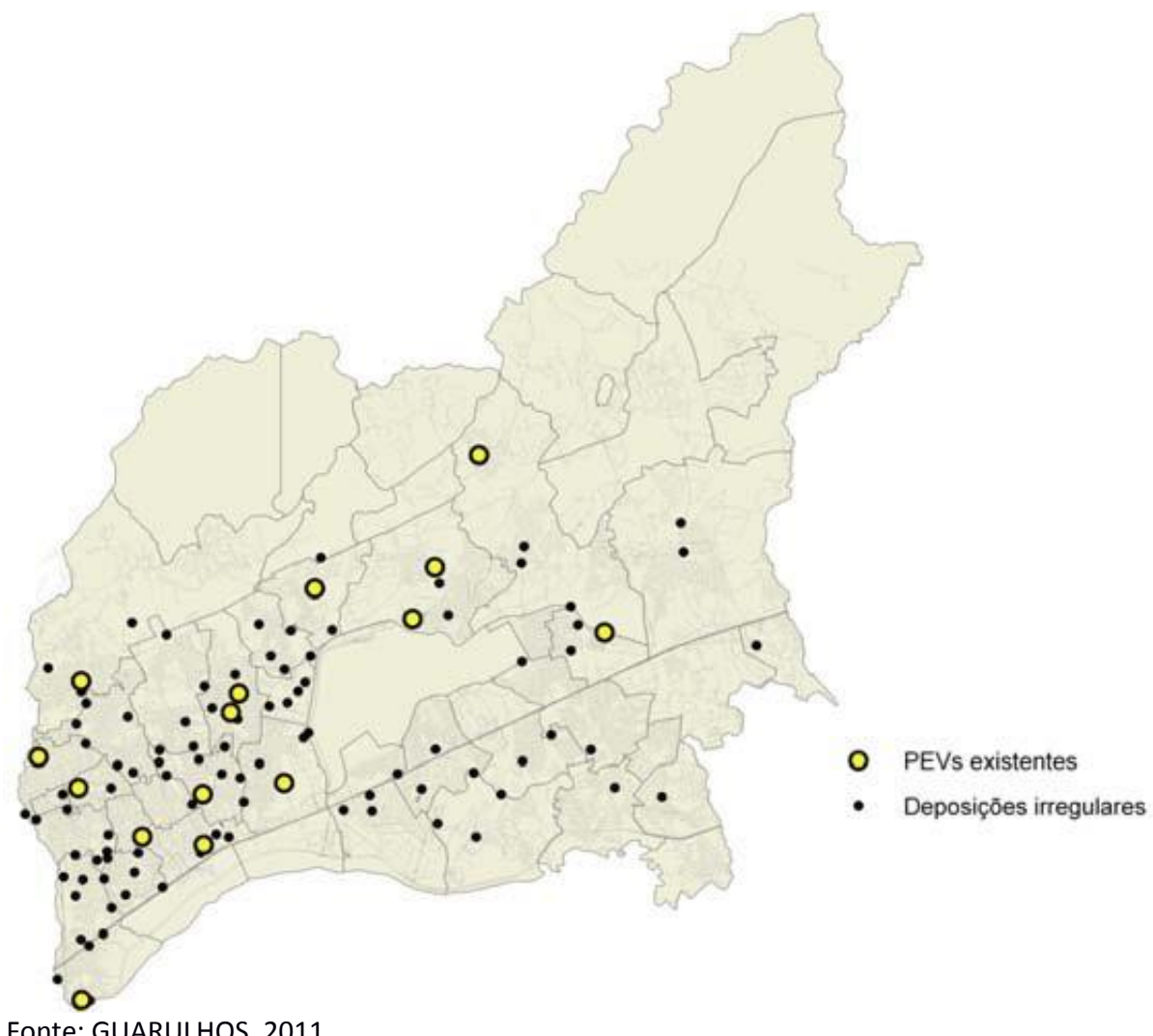

Tabela 1: Indicadores utilizados para análise espacial.

\begin{tabular}{|c|c|c|}
\hline Indicadores & Fontes & Dimensões \\
\hline $\begin{array}{c}\text { Renda média per capita por bairro } \\
(2010)\end{array}$ & $\begin{array}{c}\text { Instituto Brasileiro de Geografia e Estatística - } \\
\text { IBGE, 2010 }\end{array}$ & Sociodemográfico \\
\hline $\begin{array}{c}\text { Taxa de alfabetização por bairro } \\
\text { (2010) }\end{array}$ & $\begin{array}{c}\text { Instituto Brasileiro de Geografia e Estatística - } \\
\text { IBGE, 2010 }\end{array}$ & Sociodemográfico \\
\hline $\begin{array}{c}\text { Densidade demográfica (2010) } \\
\text { \% de domicílios com abastecimento } \\
\text { de água por rede geral (2010) }\end{array}$ & $\begin{array}{c}\text { Instituto Brasileiro de Geografia e Estatística - } \\
\text { IBGE, 2010 }\end{array}$ & Sociodemográfico \\
\hline $\begin{array}{c}\text { \% de domicílios com coleta de lixo } \\
\text { por bairro (2010) }\end{array}$ & $\begin{array}{c}\text { Instituto Brasileiro de Geografia e Estatística - } \\
\text { IBGE, 2010 }\end{array}$ & $\begin{array}{c}\text { Infraestrutura } \\
\text { Urbana }\end{array}$ \\
\hline $\begin{array}{c}\text { \% de domicílios com acesso à } \\
\text { iluminação pública por bairro (2010) }\end{array}$ & $\begin{array}{c}\text { Instituto Brasileiro de Geografia e Estatística - } \\
\text { IBGE, 2010 }\end{array}$ & $\begin{array}{c}\text { Infraestrutura } \\
\text { Urbana }\end{array}$ \\
\hline $\begin{array}{c}\text { Pontos de Entrega Voluntária por } \\
\text { bairro (2010) }\end{array}$ & $\begin{array}{c}\text { Revisão do Plano Diretor de Desenvolvimento } \\
\text { Urbano, Econômico e Social de Guarulhos - } \\
\text { Ambiens, 2012 }\end{array}$ & $\begin{array}{c}\text { Infraestrutura } \\
\text { Urbana }\end{array}$ \\
\hline $\begin{array}{c}\text { Deposições irregulares de RCC por } \\
\text { bairro (2010) }\end{array}$ & $\begin{array}{c}\text { Revisão do Plano Diretor de Desenvolvimento } \\
\text { Urbano, Econômico e Social de Guarulhos - } \\
\text { Ambiens, 2012 }\end{array}$ & $\begin{array}{c}\text { Desigualdade } \\
\text { Ambiental }\end{array}$ \\
\hline
\end{tabular}

A dependência espacial é uma característica inerente à representação de dados através de subdivisões territoriais. Ela pode ser entendida como a tendência a que o valor de uma variável associada a uma determinada localização assemelhe-se mais ao de amostras vizinhas do que 


\title{
Periódica Eletrônica
}

\section{Fórum Ambiental}

Volume 14, Número 2, 2018

\author{
da Alta Paulista
}

ao restante das localizações do conjunto amostral. A necessidade de quantificação da dependência espacial presente em um conjunto de geodados levou ao desenvolvimento da chamada estatística espacial, cuja característica principal é seu foco em inquirir padrões espaciais de lugares e valores e, assim, identificando a associação espacial existente entre eles e a variação sistemática do fenômeno por localização (ANSELIN, 1995).

O índice global de Moran é a estatística mais difundida, na determinação da dependência espacial de dados, pois determina a autocorrelação espacial a partir do produto dos desvios em relação à média, indicando, assim, o grau de associação espacial presente em um dado conjunto de dados. De forma geral, o índice global de Moran presta-se a um teste de hipóteses possibilitando a visualização da dependência espacial entre duas variáveis. Valores positivos ( 0 $a+1)$ indicam uma correlação direta e negativos (-1 a 0$)$ correlação inversa. Um resultado próximo a 0 indica uma pequena ou ausente correlação entre as variáveis.

O índice local de Moran é uma metodologia auxiliar ao índice global de Moran, e considera as dinâmicas espaciais com base na setorização dos mapas, gerando um mapa que aponta as particularidades regionais, entre as duas variáveis analisadas. Para construção deste mapa, utiliza-se coordenadas dos centróides das áreas, aproximando-as para um espaçamento regular de forma a montar uma matriz. Calcula-se, então, as médias e as medianas do indicador ao longo das linhas (eixo Leste-Oeste) e colunas (eixo Norte-Sul) desta matriz. Esta técnica permite identificar a flutuação das medidas ao longo de duas direções, sugerindo a presença de valores discrepantes quando a diferença entre estas é grande (nãoestacionariedade de segunda ordem), e a tendência (não-estacionariedade de primeira ordem), ao longo de uma direção. Quando os valores variam suavemente. Esse cálculo gera o Mapa de Espalhamento de Moran (Moran Scatterplot Map) mostrando cinco tipos de correlação por setor: Alto-Alto, indica um valor alto para primeira variável no setor e um valor alto para a segunda variável no setor e em sua vizinhança; Baixo-Alto, valor baixo para primeira variável no setor e valor alto para a segunda variável no setor e em sua vizinhança; Alto-Baixo, valor alto para primeira variável no setor e um valor baixo para a segunda variável no setor e em sua vizinhança; Baixo-Baixo, valor baixo para primeira variável no setor e um valor baixo para a segunda variável no setor e em sua vizinhança; e Não Significante, indicando que a correlação entre a primeira e a segunda variáveis não geram correlação espacial com base na valor analisado. A distribuição das correlações no Mapa de Moran possibilita a formação de aglomerações e resultados atípicos em diferentes regiões do espaço urbano, onde estes devem ser analisados de forma separada na compreensão de como suas características diferem dos setores vizinhos (DRUCK et al, 2004).

A análise estatística e elaboração do mapa de correlação local e o cálculo do índice global de Moran foi realizada utilizando-se o software-livre GeoDa 1.12.1.57. Para a análise correlacional, utilizou-se a matriz rainha de vizinhança, que diferente de outros tipos de matriz de pesos por realizar a análise em cada região, considerando como vizinhança apenas os casos nas quais duas regiões apresentam fronteiras comuns com a inicial, seja na horizontal, vertical ou diagonal. Utilizou-se o critério de vizinhança de primeira ordem, para representar a heterogeneidade de um espaço urbano, adotando-se taxa de $5 \%$ como significância estatística. 


\title{
Periódica Eletrônica
}

\section{Fórum Ambiental}

Volume 14, Número 2, 2018

\author{
da Alta Paulista
}

ISSN 1980-0827

Figura 2: Renda domiciliar nos bairros de Guarulhos, 2010.

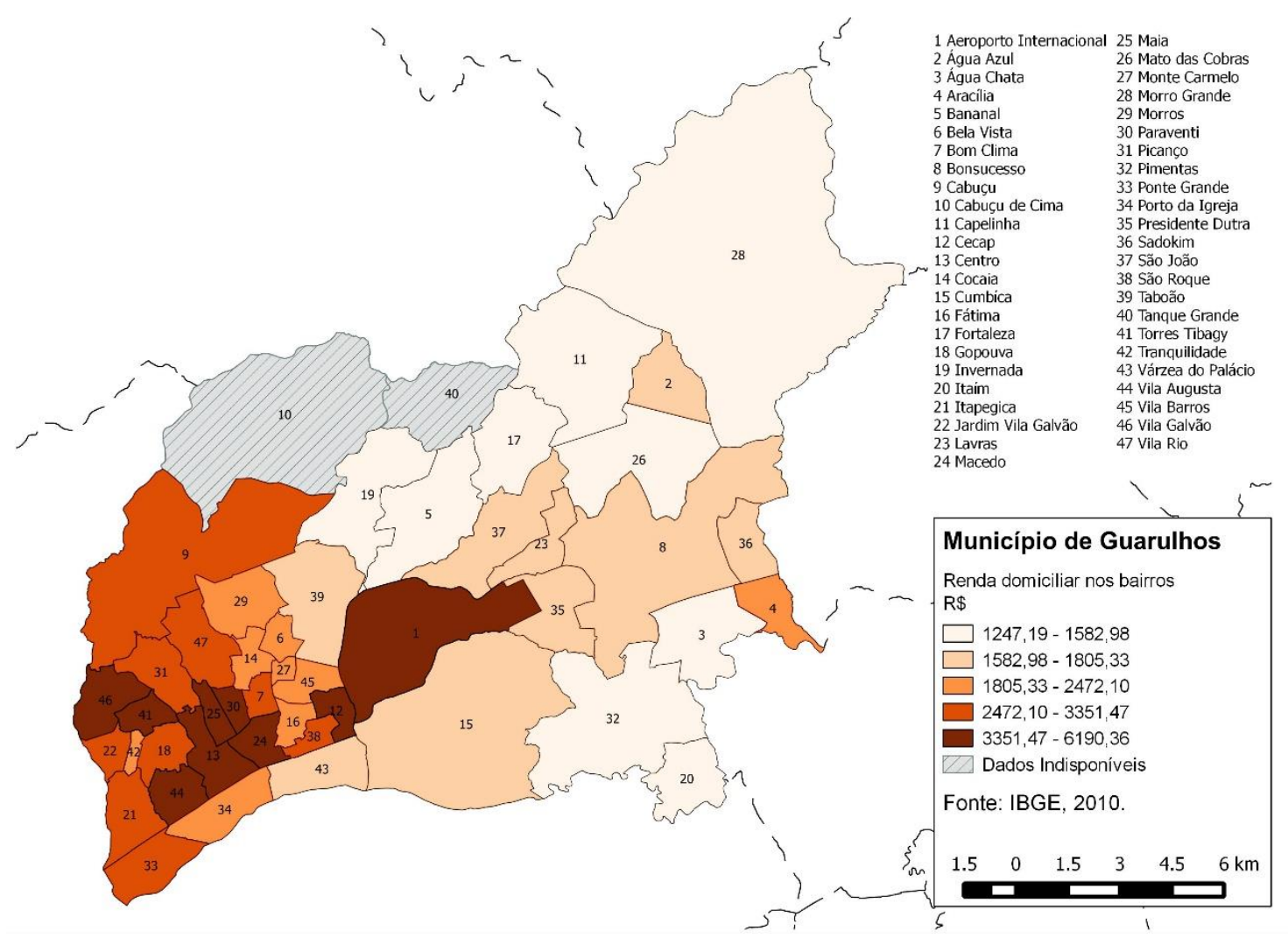

\section{RESULTADOS E DISCUSSÕES}

A diversidade de características sociais e urbanas do Município de Guarulhos fez com que se formasse aglomerados de dados sociais e de infraestrutura urbana nas mesmas regiões, sendo observado a presença de correlação baixo-baixo principalmente na região Norte do Município, e por se tratar da zona rural ou semi-urbanizada, observa-se uma menor quantidade de focos de deposição irregular de RCC e, também, os piores resultados de indicadores sociais e infraestrutura urbana. Nas regiões Central e Oeste são observados, em maior escala, as correlações alto-alto e baixo-alto, respectivamente, indicando elevados valores nos indicadores sociais e acesso à infraestrutura urbana, independente da quantidade de quantidade de pontos de descarte irregular de RCC (Fig. 3).

Em relação aos mapas locais de Moran, que divergem dos outros, o mapa referente a coleta de lixo não aponta diferenças no espaço urbano do Município de Guarulhos, sendo possível inferir que a porcentagem de domicílios que possuem coleta de lixo é similar por todo o município, com exceção da região central, onde observa-se um pequeno aglomerado baixo-alto. O mapa de PEVs indica a presença destes instrumentos públicos, especialmente nas regiões Central e Leste, onde também ocorre a presença de pontos de deposição de RCC, enquanto que, ao analisar a relação das deposições irregulares com os dados de vizinhança com outros pontos, temos o surgimento de uma região baixo-alto na região aeroportuária do município, divergindo do resto da cidade, por se tratar de uma região de uso estratégico do poder público.

O Município de Guarulhos teve uma expansão desordenada, sendo possível observar características diversas a nível social e urbano por todo o território, fazendo com que a análise 


\section{Periódica Eletroneica}

\section{Fórum Ambiental}

Volume 14, Número 2, 2018

ISSN 1980-0827

\section{da Alta Paulista}

de correlação global de Moran tenha resultados mais próximos a zero, e possibilitando que a compreensão da correlação direta ou inversa ocorra com estes valores mais baixos, com a garantia da rejeição da hipótese nula pelo grau de significância estatística adotado (Tab. 2).

Tabela 2: Análise de correlação global de Moran entre Pontos de Deposição Irregular de RCC e indicadores do município de Guarulhos

\begin{tabular}{|c|c|}
\hline Indicador & Índice Global de Moran \\
\hline Renda média per capita & 0,17 \\
\hline Alfabetização & 0,14 \\
\hline Densidade demográfica & 0,06 \\
\hline Domicílios com abastecimento de água pela rede geral & 0,16 \\
\hline Domicílios com acesso à lluminação Pública & 0,10 \\
\hline Domicílios com coleta de lixo & 0,01 \\
\hline Pontos de Entrega Voluntária & $-0,05$ \\
\hline Deposições irregulares de RCC & $-0,04$ \\
\hline
\end{tabular}

Os indicadores de renda média per capita e alfabetização, apresentaram dados semelhantes entre si, com índices positivos de correlação com os descartes irregulares. Esta similaridade pode ser atribuída a dados pertencentes aos mesmos grupos sociais. Em relação aos índices de correlação positiva com as deposições, fica evidenciado que maior grau de escolaridade e posição social não impacta de forma positiva em práticas ecológicas, como observado por JANUÁRIO et al, 2017.

A semelhança dos indicadores sociodemográficos, acesso a abastecimento de água pela rede geral e acesso à iluminação pública, indica que os investimentos destas infraestruturas ocorrem nas regiões com maior qualidade de vida, evidenciando, assim, a desigualdade social do acesso à infraestrutura pública.

O indicador domicílios com coleta de lixo mostrou índice global de Moran próximo a zero, indicando que não existe uma relação entre a coleta de lixo domiciliar e focos de descarte, podendo inferir que o lixo domiciliar não é responsável pela geração de pontos irregulares, apesar de muitas vezes se encontrarem presentes nestes espaços.

A densidade demográfica indica uma correlação positiva com o descarte irregular, evidenciando que, em áreas mais populosas, ocorre elevado número de construções e reformas que, consequentemente, contribuem para o descarte irregular dos resíduos gerados. $\mathrm{O}$ índice global de Moran, com correlação negativa de PEVs, indica que a existência destes instrumentos públicos, propicia uma maior participação da população na correta destinação do RCC e, ainda, embora muito baixo $(-0,05)$, são necessários investimentos para melhorias da destinação final dos resíduos sólidos. Isto nos sugere que há a necessidade de uma maior divulgação dos espaços de coleta e, consequentemente, a conscientização da população quanto a participação na responsabilidade compartilhada da destinação dos resíduos do Município.

A análise correlacional univariada das deposições irregulares dos bairros, ou seja, o índice que correlaciona os pontos de descarte irregulares e sua correspondência com os valores dos focos nos bairros vizinhos, indica que áreas com pontos de descartes irregulares, não necessariamente, tem em sua vizinhança bairros com pontos de descarte irregulares, evidenciando a expansão urbana desordenada e a heterogeneidade do município. 


\section{Periódica Eletroneica}

\section{Fórum Ambiental}

Volume 14, Número 2, 2018

\section{da Alta Paulista}

Figura 3: Análise de Moran Local de Deposições irregulares de resíduos da construção civil e variáveis do Município de Guarulhos. (a) Renda média per capita, (b) taxa de alfabetização, (c) densidade demográfica, (d) abastecimento de água por rede geral, (e) iluminação pública, (f) coleta de lixo, (g) pontos de entrega voluntária e (h) pontos de deposição de resíduos da construção civil.
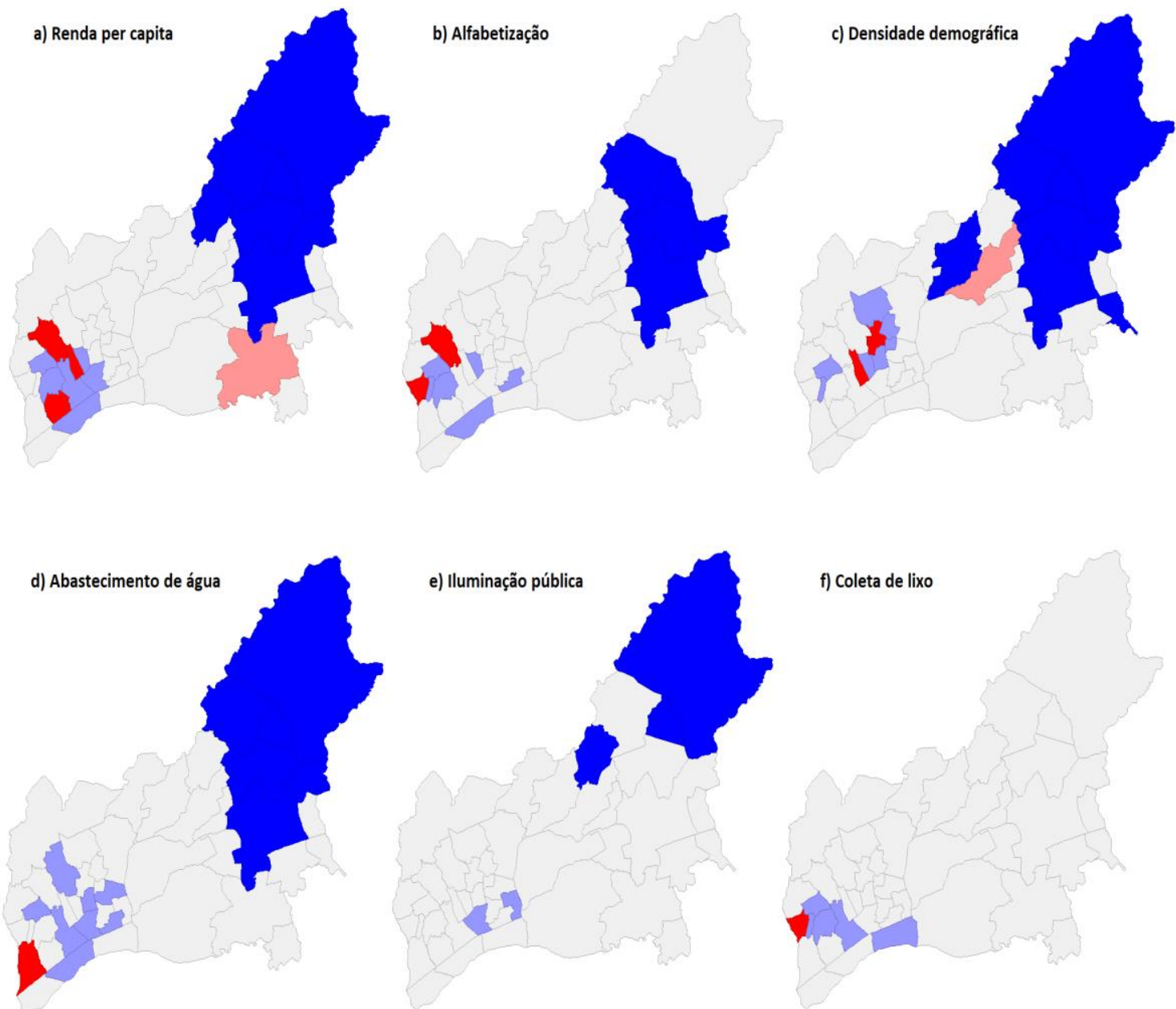

f) Coleta de lixo

g) PEVs

h) Deposição de RCC

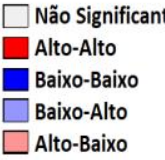




\section{Periódica Eletrônica}

\section{Fórum Ambiental}

Volume 14, Número 2, 2018

da Alta Paulista

ISSN 1980-0827

\section{CONCLUSÃO}

Primeiramente, fica evidente que o investimento público com PEVs se mostrou uma política pública que auxilia no processo da correta destinação dos resíduos da construção civil, apesar do pequeno impacto, pois nos locais onde existem os PEVs observa-se uma pequena redução do descarte irregular do RCC, podendo estar relacionado a problemas de divulgação e acessibilidade destes espaços. Podemos inferir não apenas a necessidade de expansão desses espaços, principalmente em direção às regiões mais carentes, como, também, sua maior divulgação publicitária para que haja, não apenas conhecimento da população quanto a sua existência, mas uma maior conscientização sobre sua importância.

A limpeza urbana, de acordo com os resultados obtidos na análise correlacional, indicam que seu processo de expansão possibilita, a longo prazo, a diminuição da desigualdade de acesso à urbanidade nas regiões mais afastadas.

Os resultados evidenciaram que os melhores indicadores sociais se concentram na região Central do Município de Guarulhos, coincidindo com o elevado número de pontos de descarte irregular de RCC, evidenciando a necessidade de propostas de maior atenção à coleta destes resíduos e educação ambiental, como instrumentos capazes de reduzir o impacto gerado.

A análise correlacional das variáveis possibilitou uma maior compreensão sobre a relação entre indicadores sociais e urbanos com o descarte irregular de RCC no Município de Guarulhos. Esse tipo de mapeamento possibilita a análise do impacto de indicadores oferecem dados que podem explicar potenciais fatores causadores de determinados fenômenos e serem utilizados na construção de políticas públicas mais eficientes.

\section{REFERÊNCIAS BIBLIOGRÁFICAS}

AMBIENS SOCIEDADE COOPERATIVA. Plano de Trabalho da Revisão do Plano Diretor de Desenvolvimento Urbano, Econômico e Social de Guarulhos-SP. Curitiba, 2012.

AMORIM, Fernando de Oliveira. Uma experiência de mobilização e resistência dos movimentos sociais no processo de planejamento urbano: o Projeto Nova Luz em São Paulo. 2016. Tese apresentada na Faculdade de Arquitetura e Urbanismo, Universidade de São Paulo, São Paulo, 2016.

ANSELIN, Luc. Local indicators of spatial association-LISA. Geographical analysis, v. 27, n. 2, p. 93-115, 1995.

BRASIL. Política Nacional de Resíduos Sólidos. Lei no 12.305, de 2 de agosto de 2010. Presidência da República, Departamento da Casa Civil. Brasília, 2010.

CONAMA. Resolução $\mathrm{n}$ 0 307, de 05 de julho de 2002: Estabelece diretrizes, critérios e procedimentos para a gestão dos resíduos da construção civil. Diário Oficial da União, Brasília, DF, 17 jul. 2002.

COSTA, Nébel Argüello Affonso. A reciclagem do resíduo de construção e demolição: uma aplicação da análise multivariada. Tese apresentada na Engenharia de Produção da Universidade Federal de Santa Catarina, Florianópolis, 2003.

DE LÓCCO, Ligia Goncalves. Política de limpeza urbana em Guarulhos: análise da formulação e implementação Dissertação apresentada na Escola de Artes, Ciências e Humanidades da Universidade de São Paulo, São Paulo, 2017.

DRUCK, Suzana; CARVALHO, Marilia Sá; CÂMARA, Gilberto; MONTEIRO, Antônio Miguel (Eds) (2004). Análise Espacial de Dados Geográficos. Brasília, EMBRAPA, 2004.

FERREIRA, Arildo; PROCIDONIO, Maristela; PRESTES, Sebastião Sergio. Os consórcios intermunicipais do Estado do Paraná resultantes do Plano Nacional de Gerenciamento de Resíduos Sólidos: estudo da Região 17. Anais do 8으. Fórum Internacional de Resíduos Sólidos, 2017. 


\section{Periódica Eletroneica}

\section{Fórum Ambiental}

Volume 14, Número 2, 2018

da Alta Paulista

ISSN 1980-0827

GUARULHOS. DECRETO № 25754 de 01 de setembro de 2008 que regulamenta o Plano Integrado de Gerenciamento de Resíduos da Construção Civil.

GUARULHOS. Plano Diretor de Resíduos Sólidos - PGIRS do Município de Guarulhos. Guarulhos, 2011.

IBGE. Censo Demográfico 2010 - Resultados Preliminares do Universo. Rio de Janeiro: IBGE, 2011.

JACOBI, Pedro Roberto.; BESEN, Gina Rizpah. Gestão de resíduos sólidos em São Paulo: desafios da sustentabilidade. Revista Estudos Avançados, v.15, n. 71, São Paulo, jan./abr. 2011.

JANUÁRIO, Mauro. FERNANDES, Flávia Regina Moreira. VALÉRIO, Maria Apparecida. MACEDO, Rogério Barbosa. Estudo do Comportamento Ambiental da População de Wenceslau Braz/PR em Relação aos Resíduos Sólidos Urbanos. Revista de Gestão Ambiental e Sustentabilidade, v. 6, n. 1, p. 55-71, 2017.

LINHARES, Silvia Paixão; FERREIRA, João Alberto; RITTER, Elisabeth. Avaliação da implantação da Resolução $n$. 307/2002 do CONAMA sobre gerenciamento dos resíduos de construção civil. Revista Estudos Tecnológicos em Engenharia, v. 3, n. 3, p. 176-194, 2007.

MINISTÉRIO DO MEIO AMBIENTE. Secretaria de Recursos Hídricos e Ambiente Urbano. Manual para Implantação de Sistema de Gestão de Resíduos de Construção Civil em Consórcios Públicos. Brasília, 2010.

SAAD, Antonio Roberto, SEMENSATTO JR, Décio Luis, AYRES, Fernando Martins, DE OLIVEIRA, Paulo Eduardo. Índice de Qualidade da Água-IQA do Reservatório do Tanque Grande, Município de Guarulhos, Estado de São Paulo, Brasil: 1990-2006. Revista Geociências-UnG-Ser, v. 6, n. 1, p. 118-133, 2007.

TESSARO, Alessandra Buss; DE SÁ, Jocelito Saccol; SCREMIN, Lucas Bastianello. Quantificação e classificação dos resíduos procedentes da construção civil e demolição no município de Pelotas, RS. Revista Ambiente Construído, Porto Alegre, v. 12, n. 2, p. 121-130, abr./jun. 2012.

VIÉGAS, Rodrigo Nuñez. Desigualdade Ambiental e "Zonas de Sacrifício". Rio de Janeiro: FASE/IPPUR (Artigo publicado no Mapa dos Conflitos Ambientais no estado do Rio de Janeiro), 2006. 